\title{
SARIM PLUS-sample return of comet 67P/CG and of interstellar matter
}

R. Srama • H. Krüger • T. Yamaguchi - T. Stephan • M. Burchell •

A. T. Kearsley • V. Sterken • F. Postberg • S. Kempf • E. Grün • N. Altobelli •

P. Ehrenfreund • V. Dikarev • M. Horanyi • Z. Sternovsky • J. D. Carpenter •

A. Westphal · Z. Gainsforth • A. Krabbe • J. Agarwal · H. Yano • J. Blum •

H. Henkel • J. Hillier · P. Hoppe • M. Trieloff • S. Hsu • A. Mocker •

K. Fiege • S. F. Green • A. Bischoff • F. Esposito • R. Laufer • T. W. Hyde •

G. Herdrich • S. Fasoulas • A. Jäckel • G. Jones • P. Jenniskens • E. Khalisi •

G. Moragas-Klostermeyer • F. Spahn • H. U. Keller • P. Frisch •

A. C. Levasseur-Regourd • N. Pailer • K. Altwegg • C. Engrand • S. Auer •

J. Silen • S. Sasaki • M. Kobayashi • J. Schmidt • J. Kissel • B. Marty •

P. Michel · P. Palumbo • O. Vaisberg • J. Baggaley • A. Rotundi • H. P. Röser

Received: 17 March 2011 / Accepted: 22 December 2011 / Published online: 11 February 2012 (C) Springer Science+Business Media B.V. 2012

Abstract The Stardust mission returned cometary, interplanetary and (probably) interstellar dust in 2006 to Earth that have been analysed in Earth laboratories worldwide. Results of this mission have changed our view and

\footnotetext{
R. Srama $(\varangle)$

Institut für Raumfahrtsysteme (IRS), Universität Stuttgart, Raumfahrtzentrum Baden Württemberg Pfaffenwaldring 29, 70569 Stuttgart, Germany

e-mail: srama@irs.uni-stuttgart.de

V. Sterken · F. Postberg · A. Krabbe · A. Mocker · G. Herdrich · S. Fasoulas ·

G. Moragas-Klostermeyer · H. P. Röser

University of Stuttgart, IRS, Stuttgart, Germany

R. Srama · E. Grün · A. Mocker · K. Fiege · E. Khalisi ·

G. Moragas-Klostermeyer

Max Planck Institute Nuclear Physics, Heidelberg, Germany

H. Krüger

Max Planck Institute Solar System Research, Katlenburg-Lindau, Germany

T. Yamaguchi

JAXA, Kanagawa, Japan

T. Stephan · P. Frisch

University of Chicago, Chicago, IL, USA
} 
knowledge on the early solar nebula. The Rosetta mission is on its way to land on comet 67P/Churyumov-Gerasimenko and will investigate for the first time in great detail the comet nucleus and its environment starting in 2014. Additional astronomy and planetary space missions will further contribute to our understanding of dust generation, evolution and destruction in interstellar and interplanetary space and provide constraints on solar system formation and processes that led to the origin of life on Earth. One of these missions, SARIM-PLUS, will provide a unique perspective by measuring interplanetary and interstellar dust with high accuracy and sensitivity in our inner solar system between 1 and 2 AU. SARIM-PLUS employs latest in-situ techniques for a full characterisation of individual micrometeoroids (flux, mass, charge, trajectory, composition) and collects and returns these samples to Earth for a detailed analysis. The opportunity to visit again the target comet of the Rosetta mission 67P/Churyumov-Gerasimeenternko, and to investigate its dusty environment six years after Rosetta with complementary methods is unique and strongly enhances and supports the scientific exploration of this target and the entire

M. Burchell

University of Kent, Canterbury, UK

A. Kearsley

Natural History Museum, London, UK

V. Sterken · S. Kempf · J. Blum · K. Fiege · H. U. Keller

University of Braunschweig, Braunschweig, Germany

F. Postberg · M. Trieloff

University of Heidelberg, Heidelberg, Germany

S. Kempf · E. Grün · M. Horanyi · Z. Sternovsky · S. Hsu

University of Colorado, LASP, Boulder, CO, USA

J. D. Carpenter

ESA-ESTEC, Noordwijk, The Netherlands

N. Altobelli

ESA-ESRIN, Madrid, Spain

P. Ehrenfreund · A. Westphal · Z. Gainsforth

University of California, Berkeley, CA, USA

V. Dikarev · J. Agarwal · F. Spahn · J. Schmidt

University of Potsdam, Potsdam, Germany

H. Yano

JAXA-ISAS, JSPEC, Kanagawa, Japan

H. Henkel

von Hoerner \& Sulger, Schwetzingen, Germany

J. Hillier · S. F. Green

Open University, Milton Keynes, UK 
Rosetta mission. Launch opportunities are in 2020 with a backup window starting early 2026. The comet encounter occurs in September 2021 and the reentry takes place in early 2024. An encounter speed of $6 \mathrm{~km} / \mathrm{s}$ ensures comparable results to the Stardust mission.

Keywords Interstellar dust - Cometary dust - Churyumov Gerasimenko • Interplanetary dust $\cdot \mathrm{IMF} \cdot \mathrm{Cosmic}$ vision $\cdot$ Sample return $\cdot$ Dust collector $\cdot$ Mass spectrometry

\title{
1 Science objectives
}

Dust evolution in interstellar and interplanetary space is directly related to the origin of our solar system and processes of star formation. A significant portion of cosmic dust measurements have been obtained by remote-sensing methods. Scientific discoveries demand confirmation and new research avenues and

\author{
P. Hoppe $\cdot$ A. Bischoff \\ University of Münster, Münster, Germany \\ F. Esposito $\cdot$ P. Palumbo $\cdot$ A. Rotundi \\ University Parthenope, Napoli, Italy \\ R. Laufer · T. W. Hyde \\ Baylor University, Waco, TX, USA \\ A. Jäckel · K. Altwegg \\ University Bern, Bern, Switzerland \\ G. Jones \\ University College London, London, UK \\ P. Jenniskens \\ SETI Institute, Mountain View, CA, USA
}

A. C. Levasseur-Regourd

UPMC, University Paris, Paris, France

N. Pailer

Astrium GmbH, Friedrichshafen, Germany

C. Engrand

CNRS Paris, CSNSM, Paris, France

S. Auer

A \& M Associates, Basye, VA, USA

J. Silen

FMI, Helsinki, Finland

S. Sasaki

NAOJ, Mizusawa, Japan 
alternate methods of studying cosmic dust. The SARIM PLUS mission combines in-situ analysis of interstellar, interplanetary and cometary dust and sample return.

The solar system is currently passing through a small cloud of low density weakly ionized interstellar medium of our galaxy. Twenty years ago, galactic dust traversing the planetary system has been identified by the dust detector onboard the Ulysses spacecraft [17]. Since then, analyses of data obtained at and within the Earth orbit by different spacecraft (Helios, Galileo, and Cassini) has shown that a significant amount of interstellar dust (ISD) is in our reach [2]. This observation opened the possibility for the Stardust mission [5] to make the first attempt capturing a few ten contemporary ISD grains and delivering them to the Earth where they are subject to detailed analysis.

There is compelling science to re-fly Stardust-like missions with improved instrumentation. It is proposed to collect interstellar dust particles in interplanetary space and return them to Earth. SARIM PLUS has the following advantages with respect to the collection of ISD grains:

- Optimised for the collection of interstellar dust with a ten time increased sensitive area $\left(\sim 1 \mathrm{~m}^{2}\right)$,

- Discrimination between interplanetary and interstellar dust using latest detection techniques (Dust Telescope/Orbitrap)

- Improved dust collectors of various materials, optimised for specific analysis methods,

- Identification of individual impact locations on the collector by the means of advanced Active Collectors and determination of individual particle trajectories.

Information that can be retrieved from interstellar dust samples is of great value and can in principle not be foreseen. First laboratory analyses shall focus on the isotopic composition, the inorganic and organic chemical composition and the mineralogical/ petrological composition.

\author{
M. Kobayashi \\ PERC, CIOT, Narashino City, Japan \\ J. Kissel \\ MPE, Garching, Germany \\ B. Marty \\ CRPG, Nancy, France \\ P. Michel \\ CNRS, Nice, France \\ O. Vaisberg \\ IKI-RSSI, Moscow, Russia \\ J. Baggaley \\ University of Canterbury, Christchurch, New Zealand
}


Monitoring the evolution of dust particles offers a unique opportunity to learn more about their formation, processing and destruction. The composition and optical properties of interstellar grains are important to determine, e.g. for astronomical observations and the question of how much star light is obscured by cosmic dust, which in turn determines the amount of dark matter in our universe. Now, the analysis of galactic dust with spacecraft at Earth distance from the Sun is within our reach. SARIM PLUS combines the scientific goals of DuneXpress [18] and SARIM [44].

\subsection{Local interstellar dust}

Our Solar System is currently moving through the nearby interstellar medium (ISM) which is dominated by shells of gas with low density $\left(\mathrm{n}(\mathrm{H}) \approx 0.2 \mathrm{~cm}^{-3}\right.$, [38]) (LIC, Local Interstellar Cloud). The LIC was caused by supernova explosions and belongs to a cluster of clouds originating from the direction of the associations of Scorpius-Centaurus and Orion, star-forming regions in our neighborhood. It was shown by Landgraf et al. [32] that dust particles with different sizes behave differently in the LIC: Whereas small particles couple via electromagnetic interactions to the ISM on length scales $<3$ light years, bigger grains $(>10 \mu \mathrm{m})$ couple to the gas over much longer scales (30 to 300 light years) and remain unaffected over larger distances: ISD would be expected to come directly from their source region.

Seventeen years ago, interstellar dust was identified inside our planetary system by the dust detector onboard the Ulysses spacecraft [17]. A flow of $\mu \mathrm{m}-$ sized interstellar grains has been identified at a distance of about five AU from the Sun. The observed flux was $1.5 \cdot 10^{-4} \mathrm{~m}^{-2} \mathrm{~s}^{-1}$ of particles with a mean mass of $3 \cdot 10^{-13} \mathrm{~g}$ giving a mass flux of $5 \cdot 10^{-17} \mathrm{gm}^{-2} \mathrm{~s}^{-1}$. The interstellar dust enters the Solar System with a speed of $26 \mathrm{~km}^{-1}$ and its direction of $259^{\circ}$ ecliptic longitude and $+8^{\circ}$ latitude was found to be compatible with the direction of the interstellar neutral helium gas as detected by Ulysses [32, 53]. In-situ measurement methods allowed the determination of dust masses for individual dust impacts and the detected grain sizes differed with distance to the Sun revealing a lack of small grains $(<3 \mu \mathrm{m})$ inside a heliocentric distance of $3 \mathrm{AU}[33,54]$. At distances between 0.7 and $3 \mathrm{AU}$ the Cassini and Galileo dust instruments detected only particles bigger than $0.5 \mu \mathrm{m}[1,3]$. Most recently, a reanalysis of the Helios data set, measured at 0.3 AU heliocentric distance, revealed grains even bigger than $1 \mu \mathrm{m}[2]$.

In addition to in-situ detections, ground-based radar probing has revealed the existence of $\sim 20 \mu \mathrm{m}$ sized meteoroids with interstellar trajectories in the inner solar system [4]. The precise orbits obtainable via radar probing (orbital uncertainty $\sim 2^{\circ}$ ) make the technique valuable complementary to spacecraft.

Laboratory isotopic analysis of meteorites and interplanetary dust collected in the Earth's upper atmosphere have revealed the presence of nmto micrometer-sized refractory dust grains with highly anomalous isotopic compositions. These so-called presolar grains must have formed in the winds of evolved stars or in the ejecta of stellar explosions [20]. Among the identified 
presolar minerals are diamond, silicon carbide, graphite, silicon nitride, corundum and other forms of $\mathrm{Al}_{2} \mathrm{O}_{3}$, spinel, hibonite, titanium oxide, and a variety of silicates. Many of these minerals are also observed in the atmosphere of evolved stars, as inferred from stellar spectra.

The current tools available for astronomers are remote sensing observations of the interstellar dust and the interstellar medium. Spectroscopic measurements of the absorption and emission bands are the common standard technique. UV measurements determined the characteristics of the extinction bump at around $217 \mathrm{~nm}$ to be an absorption by electronic transitions of graphite. But due to the limited access to interstellar grains, many questions are still open. Currently, compositional information is only derived from remote sensing observations and results of the Stardust mission are still in process. Can destruction and formation processes like coagulation and condensation be identified in the composition and size-distribution of interstellar grains? Are there multi-component grains and are silicates mixed-in with carbonaceous material?

\subsection{Interplanetary dust}

Interplanetary dust particles (IDP) are more abundant than ISD in our inner solar system. Therefore SARIM-PLUS will measure IDPs alongside ISD and cometary dust, and a major objective of the SARIM-PLUS mission is to distinguish and characterise individual grains by their dynamical and elemental properties (compare this section with the scientific background of the earlier proposed SARIM mission described in Srama et al. [44]. Chemical analysis (in-situ and remote) of comets, asteroids and meteorites then provide the data to link to locations of composition and thermal gradients in the solar nebula. Such links between early solar system conditions and asteroids, comets, and meteorites today can constrain our theoretical models of the formation of the solar system solid bodies [36].

Comets, which formed in the outer solar system, are the least altered objects surviving from the formation of the solar system. They can preserve some record of the processes involved in their formation and unprocessed material from the presolar molecular cloud [35]. In-situ elemental and isotopic data for small newly ejected cometary particles was gathered by the dust impact time-of-flight mass spectrometers at comets Halley and Wild 2, onboard the spacecraft VeGa 1, VeGa 2, Giotto, and Stardust, respectively. It was found that the abundances of elements that are more refractory than oxygen appear to reflect solar composition, as is found for $\mathrm{C} 1$ chondrite meteorites. Particles rich in $\mathrm{H}, \mathrm{C}$, and $\mathrm{N}$ however, are less depleted in these elements than in $\mathrm{C} 1$ chondrites, and hence must be more primitive. Silicates form the most significant part of the refractory component in comets. Analysis of dust from comet Wild 2 by the Stardust mission $[6,34,54]$ demonstrates that cometary silicates are a mix of crystalline high-temperature grains (enstatite and forsterite) and glassy amorphous grains. The comet was made of a highly heterogeneous mixture of both minerals and organics that are far from being in chemical and 
mineralogical equilibrium. Both highly volatile and highly refractory components were in intimate contact and mixed on micron size scales. Some of these phases showed clear isotopic evidence for presolar materials. These materials were subjected to very little processing since they were accumulated into the cometary parent body. Thus, cometary particles likely represent the best samples of pristine early solar system material, and their study may provide powerful insights into the formation of the entire solar system, not just comets.

The high temperature crystalline grains found in the Stardust samples (including calcium-aluminium-inclusions, or CAIs) must have been formed in the inner solar nebula and subsequently transported (by radial mixing) to the comet formation zone or they may be true stardust formed in the atmosphere of another star [51]. The amorphous grains however, appear to constitute a major fraction of non-crystalline silicates of interstellar origin. This shows that comets contain materials that formed in a wide variety of conditions that likely spanned the entire solar nebula, as well as presolar interstellar and circumstellar environments, confirming that comets are repositories of primordial solar system materials.

The light scattering properties of the dust in the coma of comet 67P/CG have been studied through polarimetric imaging techniques, in a joint program between France and India from December 2008 to May 2009. This epoch was encompassing the last perihelion return of the comet, before its Rosetta spacecraft rendezvous in 2014. Observations suggest the presence of large slowly-moving dark dust particles before perihelion, and still immediately after it. Soon after perihelion, an increase in both intensity and polarization is a clue to an outburst, with freshly ejected sub-micron sized dust grains, possibly forming fluffy aggregates. A strong seasonal effect, related to the obliquity of the nucleus, is suspected, with large grains from the northern hemisphere before perihelion and small grains from the southern hemisphere after. These imaging results should be used to improve the strategy of the Rosetta lander release and the in-situ analysis of the dust by experiments on-board the Rosetta orbiter. It would be of major interest to complement them, in the future years, by a collection of dust samples.

\section{Science goals}

The knowledge about interstellar dust is based on results of remote sensing observations and is therefore limited. Much more information can be retrieved by in-situ or even sample return of interstellar grains. SARIM-PLUS performs both methods and addresses the following key questions related to interstellar dust:

- Can we identify original stardust in the interstellar medium?

- What are the elemental composition and the isotopic signatures of grains and their variability?

- What is the nature of carbonaceous dust and of organic precursor molecules for life? 
- What is the composition of silicate grains? Can we identify SiC in ISD particles and where is the iron?

- What are the sizes of compositionally different grains? Do we find nanodiamonds?

- What is the metallicity of the local interstellar medium?

- Can destruction and formation processes (coagulation, condensation) be identified in the composition and size-distribution of ISD?

- Are there multi-component grains? Are silicates mixed-in with carbonaceous material?

- Can we distinguish original stardust grains from dust grains that have been homogenized through processing in the ISM?

- What is the size distribution of interstellar dust at 1.5 AU and how much varies the flow direction and its dispersion with particle size? How much time-variable is the interstellar dust flow of various sizes?

- What is the shape and density of interstellar dust? Can we confirm the core-mantle model of the grains? Do we find fluffy particles? What are the optical properties of the grains?

- What are the flow directions of detected ISDs and can we infer the solar system entry directions?

Questions related to cometary and interplanetary dust are:

- What is the ratio of cometary versus asteroidal particles at 1.5 AU?

- How much is dust from comets chemically different to dust from asteroids?

- What is the detailed composition and mineralogy of cometary dust?

- What are the orbital characteristics of different types of cometary and asteroidal particles at $1.5 \mathrm{AU}$ ?

- What were the physical and chemical conditions in the proto-solar nebula?

- How complete was the chemical, isotopic, and mineralogical homogenization and what role played mixing of material in the proto-solar nebula?

- How was the interstellar material that formed the solar system metamorphosed into solar system compounds, in particular organics and minerals?

- Is today's interstellar material different from the ancient $4.6 \mathrm{Gyr}$ old material incorporated in comets and asteroids?

- Which part of presolar material is in interplanetary and cometary dust?

- What is the inventory of pristine interstellar material in comets?

- Does the chemical composition of a comet reflect its formation zone in the solar nebula and how?

- Do comets harbor the precursors of biological molecules?

- Did comets supply a significant fraction of the terrestrial organics?

- Are there large organic molecules in cometary and asteroidal dust that can be precursors of life?

- What are the dust charging mechanisms?

The SARIM-PLUS mission will carry an instrument payload consisting of dust detectors capable to characterise the dust grain properties by in-situ and 
collector/sample return technologies. This enables the mission to address the following scientific objectives:

- Characterise the interstellar dust flow through the planetary system.

- Determine the composition of interstellar grains passing through the solar system.

- Determine the composition of cometary grains in the inner coma of a comet.

- Determine the size distribution of interstellar dust at approximately $1.5 \mathrm{AU}$ distance from the sun.

- Establish the interrelation of interplanetary dust with comets, asteroids, and interstellar dust.

- Determine the composition of interplanetary grains.

- Analyse dust charging effects under solar wind conditions and determine optical grain properties.

In order to collect and measure interstellar dust in the inner solar system, the interstellar particles have to penetrate the heliosphere to reach distances as close as $1 \mathrm{AU}$. The motion of interstellar grains through the solar system is parallel to the flow of neutral interstellar hydrogen and helium gas. However, many grains are deflected by radiation pressure forces due to their optical properties. Nevertheless interstellar dust was identified by in-situ measurements as close as $0.3 \mathrm{AU}$ from the sun [2]! The radii of clearly identified grains range from 0.1 to $1 \mu \mathrm{m}$ with a maximum at about $0.3 \mu \mathrm{m}$. The payload instruments are optimised for this particle size range accordingly. The in-situ instruments will analyse the dust flux, size distribution, dust trajectories (dynamics and origin) and coarse elemental composition. They will also study time variability of the interstellar dust stream through the heliosphere and its coupling to the solar cycle.

Of highest scientific interest are compositional questions related to interstellar and cometary dust like Which form and isotopic composition does the carbon have? Are CAIs, chondrules, GEMS and nano-diamonds found? What is the abundance of enstatite and forsterite? Are there hydrated minerals? Do we find signatures of dust processing by shocks or accretion? What is the $D / H$ and ${ }^{3} \mathrm{He} /{ }^{4} \mathrm{He}$ ratio?

But the answer to those questions requires return of interstellar and cometary dust and advanced laboratory methods for their investigation.

\subsection{Limitations of Stardust samples}

The Stardust aerogel collectors very successfully collected dust particles from the coma of comet Wild 2 [49]. The samples, however, suffer from several important limitations. The particles struck the Stardust aerogel at $\sim 6.1 \mathrm{~km} \mathrm{~s}^{-1}$. At this velocity, some of the impacting material was destroyed, some was altered and some survived unaltered [6, 21, 37, 46, 54]. This greatly complicates the interpretation of data obtained from samples extracted from the aerogel. It is not always clear whether sample compositions and structures are characteristic of material original to the comet, were altered from their 
original forms, or contain processed aerogel collector material. This makes it difficult to ascertain the relative abundances of different cometary materials since there has likely been selective loss/alteration of some components over others. For example, the impact process probably selected against pristine survival of many organics [37]. Thus, while we will continue to learn more about comets from the Stardust samples, these samples will never allow us to address a number of key questions about cometary and early solar system materials. These include question like (I) What is the relative proportion of minerals and organics? (II) Are volatile organics present? (III) Are nebular condensate amorphous silicates present? The latter question is difficult to address with Stardust samples collected in aerogel because the aerogel itself is an amorphous silicate.

\subsection{Unanswered questions after Rosetta}

The Rosetta spacecraft will arrive at comet 67P/Churyumov-Gerasimenko and land on the surface of the nucleus in late 2014. The cometary nucleus will be investigated from orbit and also by a suite of instruments on board the lander. The physical characteristics of the nucleus will be investigated, its chemical composition will be analysed, and the physics of its activity examined. This will undoubtedly be a major step forward in our understanding of the chemical and physical processes relevant to the evolution of cometary nuclei. However, the analytical precision of measurements that can be obtained in modern Earth-based laboratories of returned cometary dust particles exceeds that of any in-situ instrument onboard spacecraft like Rosetta. By measuring interstellar and cometary particles in-situ and bringing them back to Earth more information can be obtained on how interstellar (molecular cloud) material (organics and minerals) metamorphosed into solar system compounds. The detailed investigation of specific molecules and their isotopic composition in Earth laboratories from captured particles will provide clues how comets may contribute to the development of life on Earth. Addressing such questions with state-of-the-art laboratory techniques has successfully been demonstrated on meteoritic samples from asteroids, the Moon, Mars and on Stardust samples. In addition to the analysis of dust grains in the cometary coma, the samples of cometary dust to be returned with the SARIM PLUS dust collectors will be available for investigations with the latest analysis techniques available in laboratories worldwide.

\section{Mission profile}

\subsection{Baseline scenario}

The SARIM PLUS mission profile is determined by the overall orbital parameters of the comet 67P/CG. These parameters are given as follows: aphelion distance is $5.72 \mathrm{AU}$, perihelion distance $1.29 \mathrm{AU}$, eccentricity 0.63 
and inclination of $7.1^{\circ}$. The next periapse occurs on august 13 in 2015 with an orbital period of 6.57 years. The overall profile consists of the launch, an interstellar/interplanetary dust collection campaign, the cometary encounter phase, a further interplanetary/interstellar dust collection phase and, finally, the reentry. Furthermore, there exist an opportunity for an extended mission.

The mission requires moderate relative impact velocities for interstellar grains $(<20 \mathrm{~km} / \mathrm{s})$ and an overall collection phase of approximately one year, and a cometary encounter within 1.5 AU around periapse. The comet encounter speed should be below $10 \mathrm{~km} / \mathrm{s}$ and the reentry speed as low as possible. The dry mass of the probe shall be more than $1200 \mathrm{~kg}$ for a launch window in the frame of 2020 to 2022. Under these assumptions various scenarios were studied and launch windows were found by T. Yamaguchi (Grad. Univ. for adv. Studies, Kanagawa) with optimal conditions for a launch on 8. Jan 2020 with a mission duration of $4 \mathrm{y}$ and the parameters of Table 1 .

These parameters do not include any margins (mass, delta-v). Furthermore, it has to be noted, that $67 \mathrm{P} / \mathrm{CG}$ changes its orbital elements during a Jupiter encounter in november 2018 and this change had to be taken into account. The SARIM PLUS trajectory is shown in Fig. 1 with the orbits of Mercury, Venus, Earth and Mars (orange). The orbit of 67P/CG is indicated in green colour. This trajectory gives a radial profile as shown in Fig. 2. The S/CEarth distance reaches its maximum at around 3 AU which determines the communication parameters. The S/C-Sun distance only slightly exceeds 2 AU allowing moderate sized solar cells.

Interstellar collection Relative impact velocities for interstellar dust is plotted in Fig. 3. The first interstellar ISD collection campaign is performed in the first half of 2021 before SARIM PLUS finishes its first loop and before the cometary encounter occurs in Sep 2021. During this phase, the relative impact speed of ISD grains is below $20 \mathrm{~km} / \mathrm{s}$ with a minimum around $6 \mathrm{~km} / \mathrm{s}$ which allows the capture of grains. Furthermore, in-situ characterisation of ISD and IDPs is possible during the entire cruise phase. High relative impact velocities allow a detailed compositional analysis using in-situ spectrometer technologies. Impact velocities as high as 40 or $50 \mathrm{~km} / \mathrm{s}$ provide excellent mass spectra and are a complementary data set to medium or low impact

Table 1 Baseline mission parameters

\begin{tabular}{ll}
\hline Earth escape & $2020 / 1 / 8$ \\
Delta-v apog raise & $683 \mathrm{~m} / \mathrm{s}$ \\
Earth depart & $1634 \mathrm{~m} / \mathrm{s}$ \\
DSM & $260 \mathrm{~m} / \mathrm{s}$ \\
Total & $2537 \mathrm{~m} / \mathrm{s}$ \\
Mass wet & $3070 \mathrm{~kg}$ in $\mathrm{GTO}$ \\
Mass dry & $1342 \mathrm{~kg}$ \\
Comet encounter & $2021 / 9 / 20$ \\
Comet enc. speed & $6.07 \mathrm{~km} / \mathrm{s}$ \\
Earth Return & $2024 / 1 / 8$ \\
Flight duration & $621 \mathrm{~d} \mathrm{out}, 840 \mathrm{~d}$ in \\
Total duration & $1461 \mathrm{~d}(4 \mathrm{y})$ \\
\hline
\end{tabular}




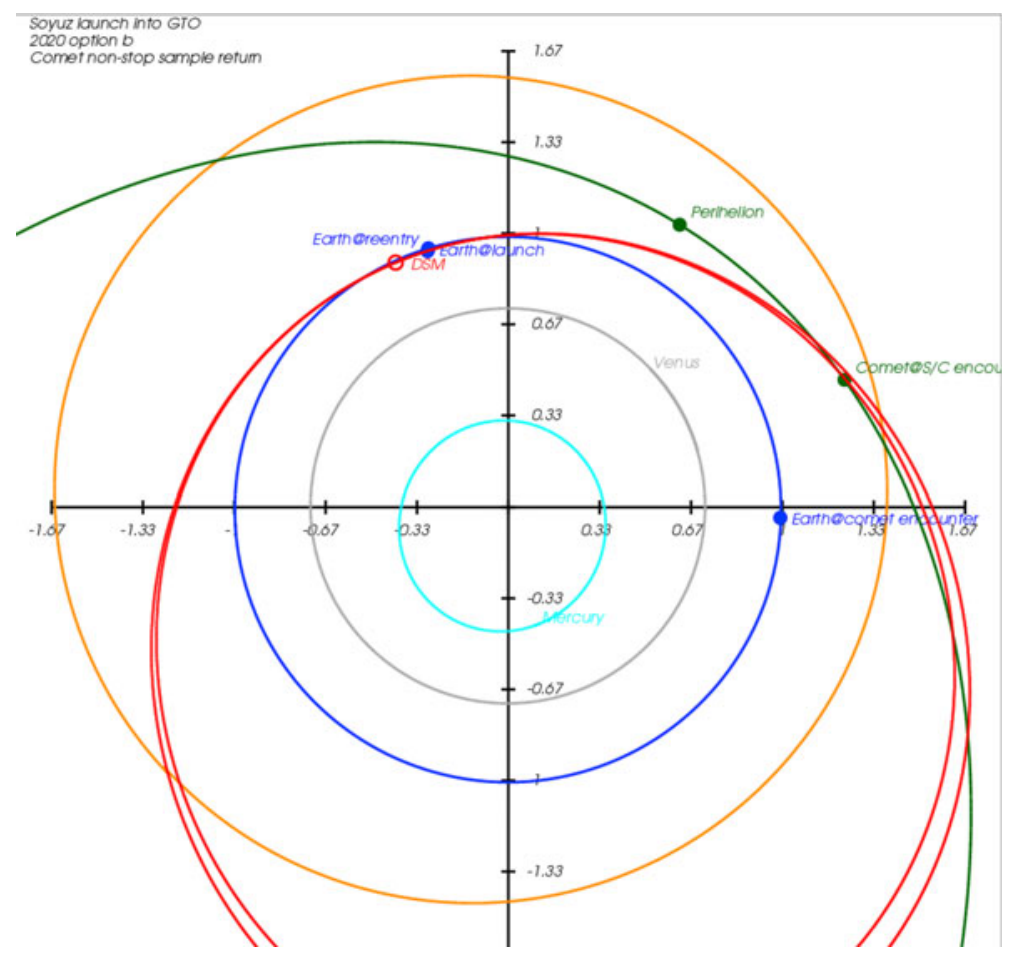

Fig. 1 Trajectory of SARIM PLUS (red) and 67P/CG (green) with a launch in 2020 and the encounter in 2021

velocities. Laboratory calibration is possible for the entire impact speed range. Generally, dust collection occurs at low impacts speeds whereas in-situ analysis is performed for the entire speed range. However, as formerly investigated in the DunXpress mission concept, the relative impact directions change and articulated collectors and solar cells are recommended. A second ISD collection phase under equal conditions is performed one year later between the deep space manoeuvre and the Earth return.

Comet encounter phase The flyby geometry of SARIM-PLUS at the comet $67 \mathrm{P} / \mathrm{CG}$ is shown in Fig. 4. The flyby distance and exact position is not decided yet and will be defined later after the Rosetta results. As a baseline, a Stardust-like scenario can be assumed. Both missions have the same flyby speed (Stardust $6.1 \mathrm{~km} / \mathrm{s}$ for Wild2, SARIM-PLUS 6.07 for $67 \mathrm{P} / \mathrm{CG}$ ) such that the operations and dust collection of Stardust will be applied to SARIMPLUS. The encounter occurs 43 days before perihelion and the distance between Earth and the space probe is only $0.56 \mathrm{AU}$ which allows high data rates and nominal power generation (Sun distance of $1.32 \mathrm{AU}$ ). No obstacles were found during the mission critical phases like the encounter or DSMs (occultations/conjunctions). 


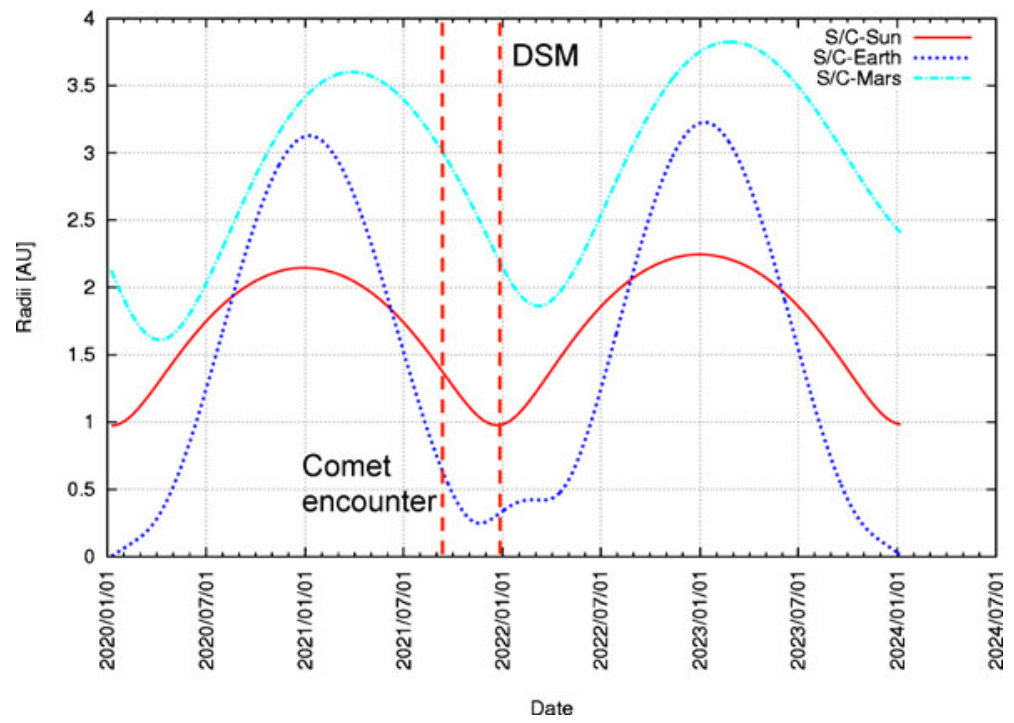

Fig. 2 Radial distance to the Sun (red line) and the Earth (dotted blue line) assuming a launch in 2020. A deep space manoeuvre (DSM) occurs in December 2021

Different types of dust collectors are exposed to the environment during the encounter phase and the plasma environment is measured simultaneously by a plasma monitor. In-situ dust measurements of the dust flux, dust mass distribution and high-resolution grain composition complement the later

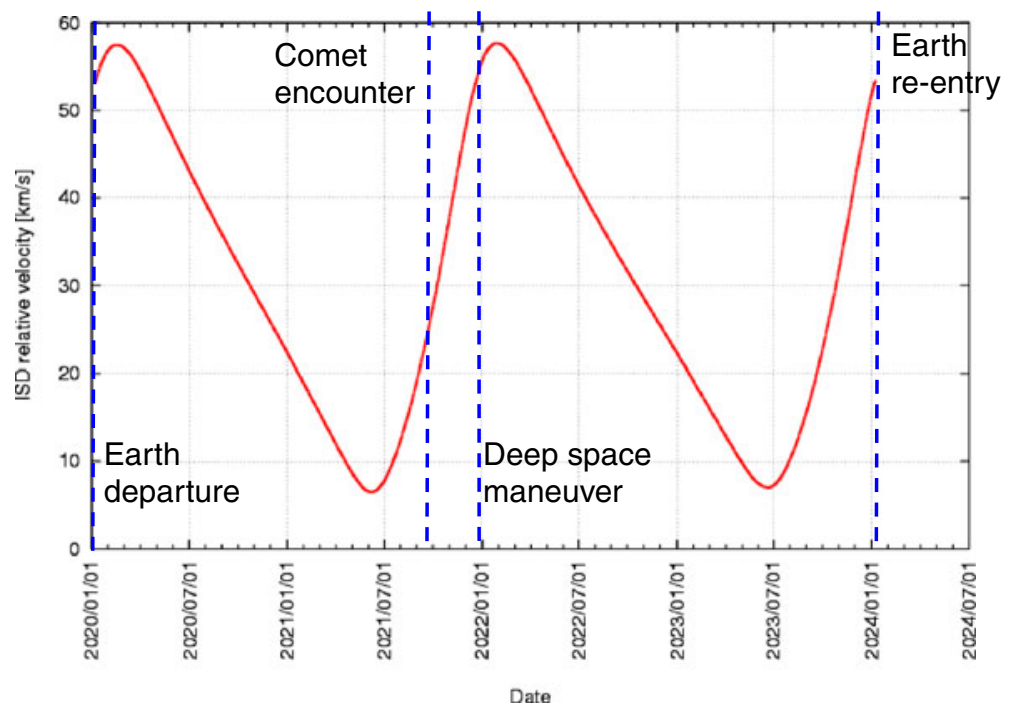

Fig. 3 Relative impact speeds of ISD. Dust is collected around the two minima 
Fig. 4 Cometary encounter geometry on Sep 20, 2021. The relative encounter speed is $6.07 \mathrm{~km} / \mathrm{s}$

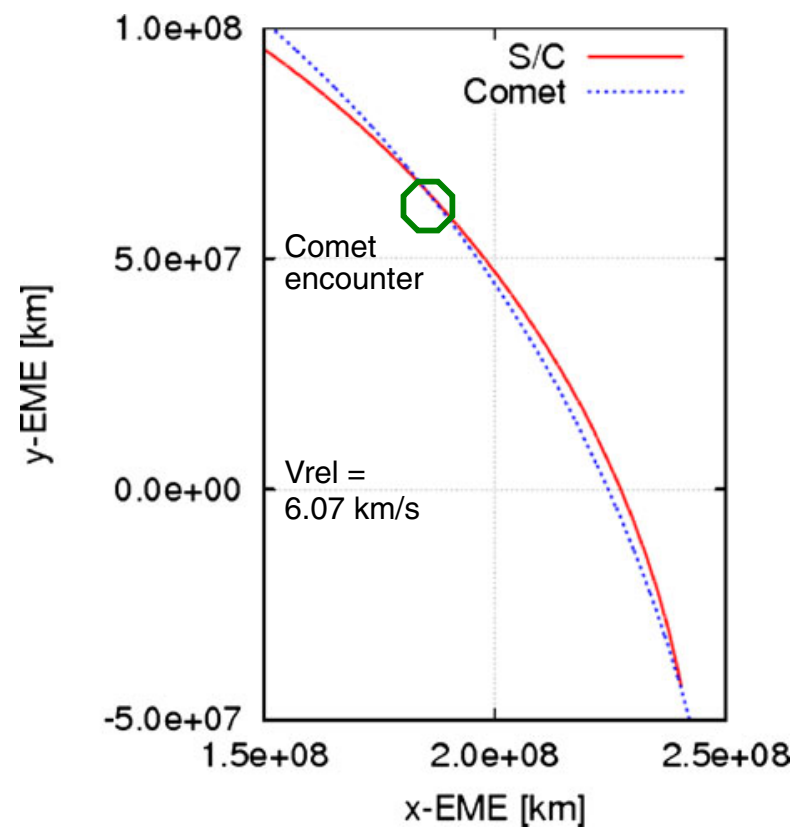

laboratory analysis of returned samples and remote sensing observations of the cometary coma. A camera will track the comet and its activity from far and close distances. The phase angles around the approach phase are shown in Fig. 5.

Reentry The return to Earth takes more than two years with further ISD and IDP collection campaigns as described above. The collectors are individual articulated modules such that different collectors are exposed during different collection phases. Reentry occurs in January 2024 with a relative speed between 11.75 and $12.68 \mathrm{~km} / \mathrm{s}$ depending on the exact conditions. This reentry speed is similar to the Stardust return speed of $12.4 \mathrm{~km} / \mathrm{s}$. Both, night or

Fig. 5 Phase angles around closest approach at $67 \mathrm{P} / \mathrm{CG}$. The Sun aspect angle is the angle between the Sun direction and the spacecraft velocity vector with respect to the comet nucleus at the time of encounter

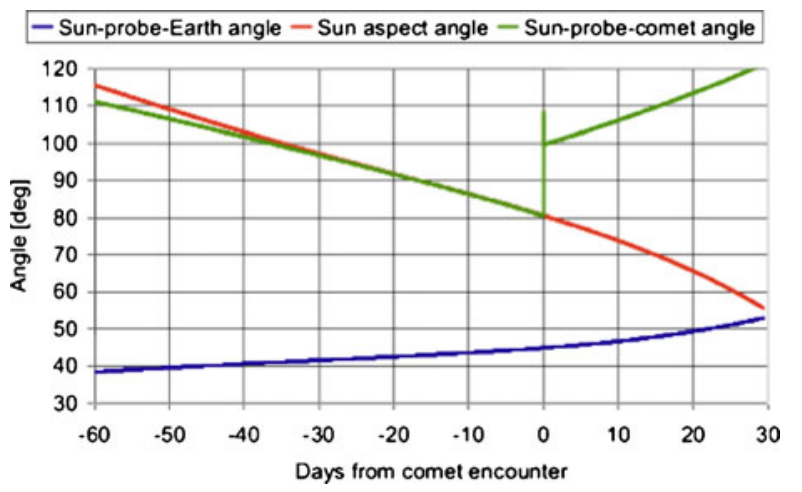




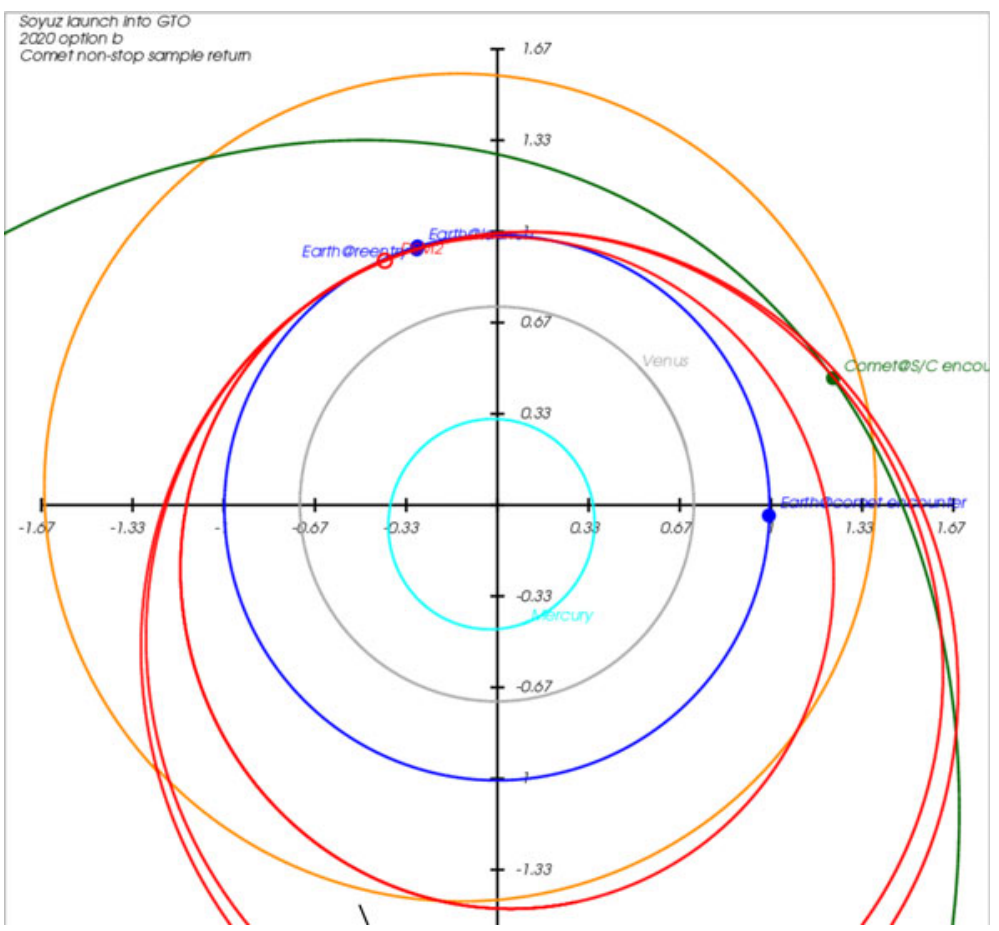

Fig. 6 SARIM PLUS extended mission trajectory after capsule separation in January 2024 (smaller red ellipse) with an aphelion close to the orbit of Mars

morning entry are available and the sites Woomera (136 E, 31 S) and Utah (114 $\mathrm{W}, 40 \mathrm{~N}$ ) are reachable. After capsule separation SARIM-PLUS can enter an extended mission with a trajectory as shown in Fig. 6. The aphelion is now smaller and just crosses the Mars orbit. As described in the former DUNE and SARIM proposals such an orbit is well suited for further in-situ IDP, ISD or nanodust investigations. The apocenter of all the orbits are directed towards the inflow (upstream direction) of the interstellar dust which enters from $8^{\circ} / 259^{\circ}$ ecliptic latitude and longitude. This leads to the measurement of higher ISD fluxes due to the mitigated influence of the IMF and the solar radiation pressure.

Table 2 Backup mission parameters

\begin{tabular}{llll}
\hline Backup profiles & $2026 \mathrm{a}$ & $2026 \mathrm{~d}$ & $2027 \mathrm{~b}$ \\
\hline Earth depart. & 2026.4 .10 & 2026.3 .25 & 2027.2 .7 \\
Comet encounter & 2028.3 .8 & 2028.3 .3 & 2028.1 .27 \\
Earth arrival & 2030.4 .10 & 2030.3 .26 & 2030.2 .7 \\
Total flight time $[\mathrm{yr}]$ & 4.00 & 4.00 & 3.00 \\
v encounter $[\mathrm{km} / \mathrm{s}]$ & 9.70 & 10.18 & 10.13 \\
V arrival $[\mathrm{km} / \mathrm{s}]$ & 3.71 & 3.68 & 4.01 \\
Dry mass $[\mathrm{kg}]$ & 1219 & 1269 & 1564 \\
\hline
\end{tabular}


A similar backup trajectory and mission scenario is hard to find because the 67P/CG perihelion passage in Nov 2021 does not allow similar solutions within the next years. Therefore the next launch window opens in early 2026 and the backup scenarios are given in Table 2.

In summary, a round-trip trajectory to $67 \mathrm{P} / \mathrm{CG}$ is found with a feasible amount of delta-v. The spacecraft will encounter the comet with a relative flyby speed of $6.1 \mathrm{~km} / \mathrm{s} 43$ days before its perihelion.

\section{Interstellar dust collection}

The interstellar dust flux over time during the SARIM mission is shown in Fig. 7. The predictions were made by deriving densities and fluxes from simulations of dust trajectories through the solar system. The flux in the graph is relative to the flux at a distance far away from the Sun, before the trajectories are disturbed by the interaction of the charged particles with the Interplanetary

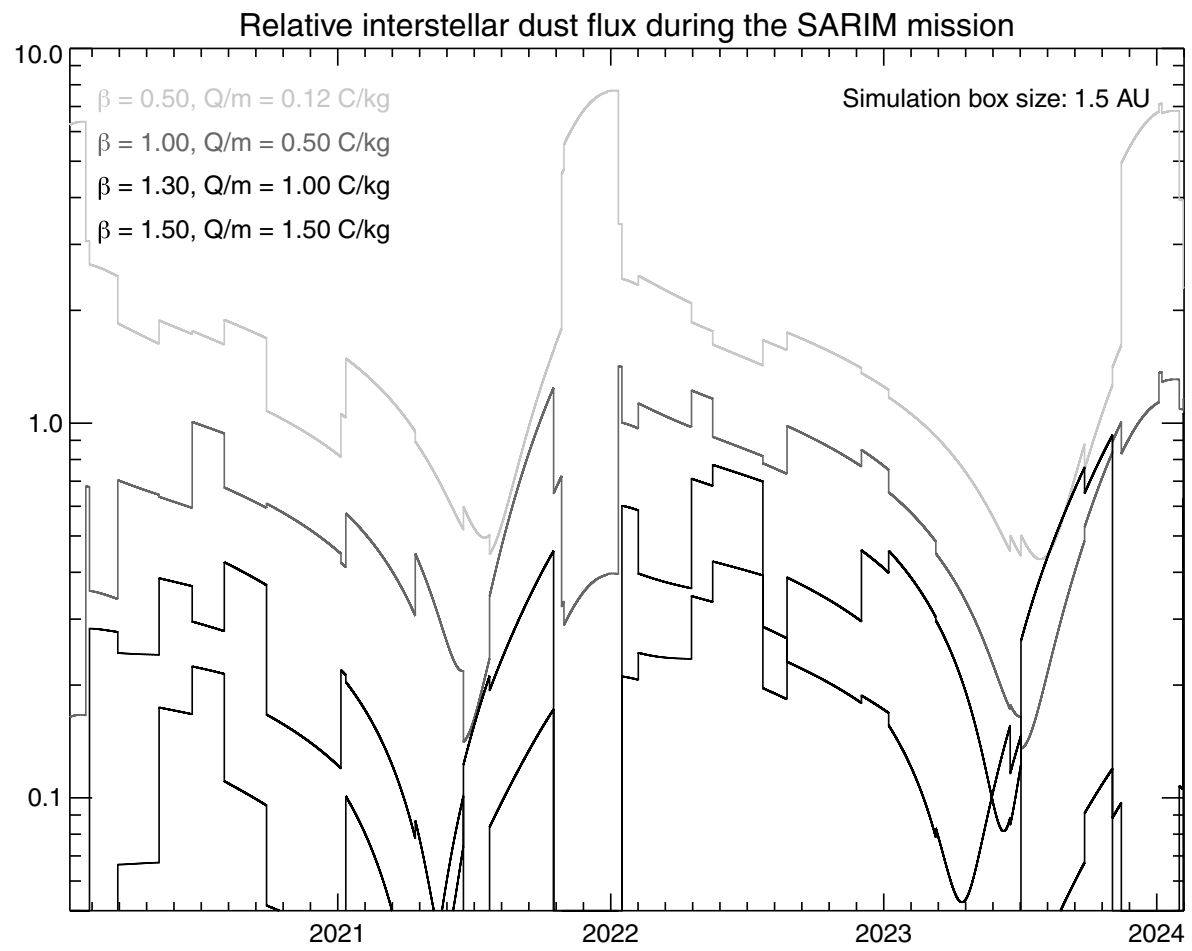

Fig. 7 Relative interstellar dust flux with respect to. infinity along the trajectory of SARIM PLUS in the years 2021 to 2024 according to the model of V. Sterken using particle sizes of $0.7,0.4,0.3$ and $0.2 \mu \mathrm{m}$ [45]. This plot shows the filtering of the heliosphere and the peak in 2022 corresponds to the focusing of large grains behind the sun. An extended mission in a focusing solar cycle would provide higher relative fluxes (best conditions are around 2030). Absolute dust fluxes cannot be derived from this plot directly. The curve steps are caused by the coarse spatial integration grid 
Table 3 Particle sizes and corresponding parameters $\beta$ and $\mathrm{Q} / \mathrm{m}$

\begin{tabular}{lll}
\hline Particle size $(\mu \mathrm{m})$ & $\beta$ & $\mathrm{Q} / \mathrm{m}(\mathrm{C} / \mathrm{kg})$ \\
\hline 0,7 & 0,5 & 0,125 \\
0,4 & 1,0 & 0,5 \\
0,3 & 1,3 & 1,0 \\
0,2 & 1,5 & 1,5 \\
\hline
\end{tabular}

Magnetic Field (IMF). The particle sizes corresponding to the flux predictions are summarized in Table 3, together with the two main particle parameters that determine the particles motion $(\beta, \mathrm{Q} / \mathrm{m}) . \beta$ is the ratio of solar radiation pressure force to solar gravity. It mainly depends on the material properties (absorption) and the size of the particle. $\mathrm{Q} / \mathrm{m}$ is the dust particle charge-tomass ratio, assuming that the grains carry a potential of $+5 \mathrm{~V}$. The chargeto-mass ratio increases with decreasing particle size such that small grains are more affected by Lorentz forces in the IMF.

The predictions in Fig. 7 show that for particles with $\beta<1$, there is a concentration of ISD downstream of the Sun due to gravitational focusing. Therefore in the years 2020, 2022, and 2024, SARIM PLUS would be able to observe considerably more of the larger particles than in the other phases of the mission. However, this would imply that SARIM PLUS points at directions close

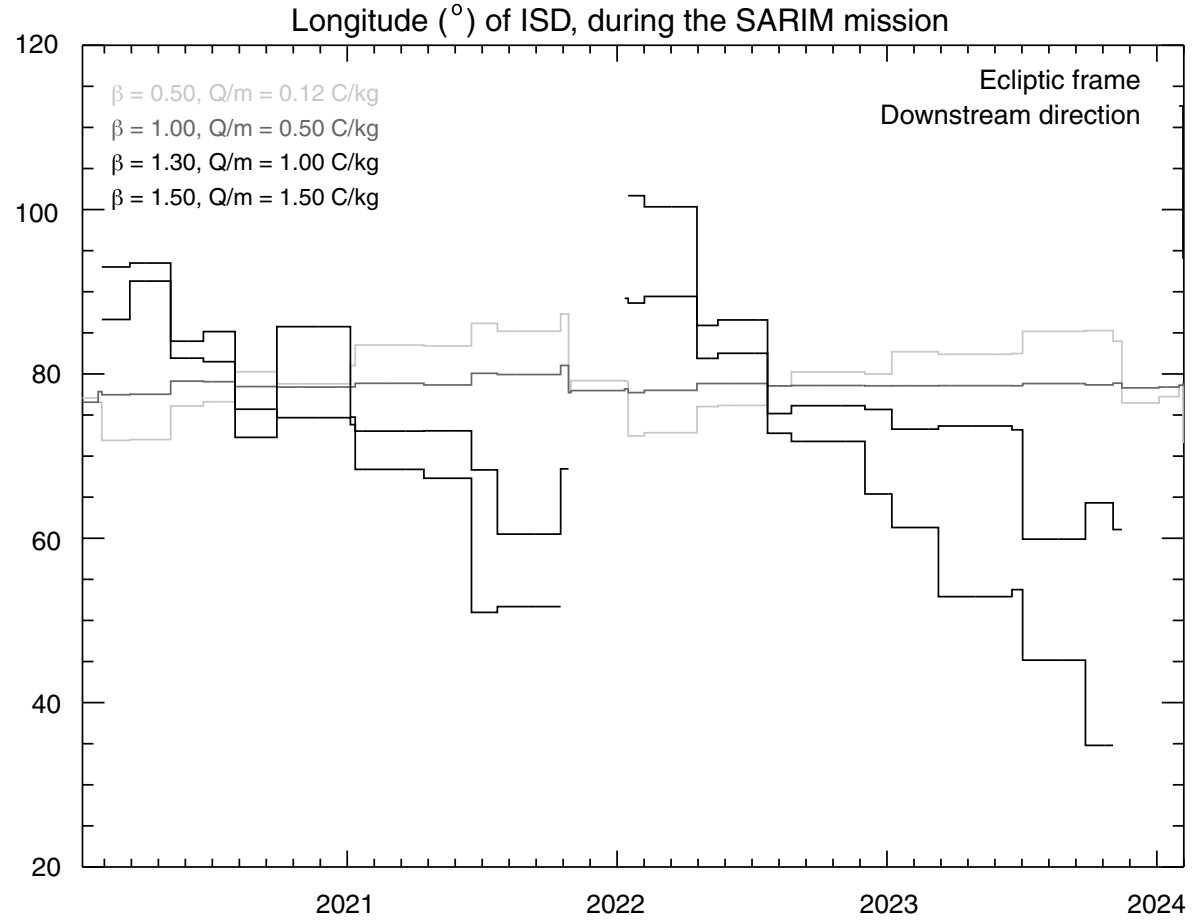

Fig. 8 Directionality variation (ecliptic longitude) of the interstellar dust direction of SARIM PLUS. Only small particles show deviations greater than $20^{\circ}$ from the interstellar longitude of $79^{\circ}$ (downstream) 
to the Sun, which is not always favorable for instrument operation reasons. Directionality predictions are used to determine the number of large particles still observed during passes of high-concentration regions. ISD flux modeling shows strong variations of grains with a $\mathrm{Q} / \mathrm{m}$ value of 0.1 and a $\beta$ value of 0.5 . Here, flux variations up to factor of 8 are possible within the year 2021 .

For particles with $\beta>1$, there is a depletion downstream from the Sun, since the solar radiation pressure force is larger than the solar gravitational force. This void region is sometimes referred to as the " $\beta$-cone", as it is paraboloidshaped and its size depends on the $\beta$-parameter of the dust. With SARIM PLUS, information about the particle size and composition will be deduced by measuring the flux through time, and relating this to the position of the $\beta$ cones, as has been done with Ulysses (" $\beta$-spectroscopy"). In Fig. 7 the $\beta$-cone is visible in 2022 through a depletion of particles. However, the width of the cone does not vary between different $\beta$-values since the grid size of the simulation used is very large (1.5 AU). Particles with higher $\mathrm{Q} / \mathrm{m}$ ratios (small particles) are predicted to be less abundant due to the interaction with the IMF.

Apart from ISD flux measurements, the SARIM PLUS capability to measure the directionality of the flux will be an extra tool for doing $\beta$-spectroscopy. During the passages through the $\beta$-gaps, information about particle properties

Latitude $\left(^{\circ}\right)$ of ISD, during the SARIM mission

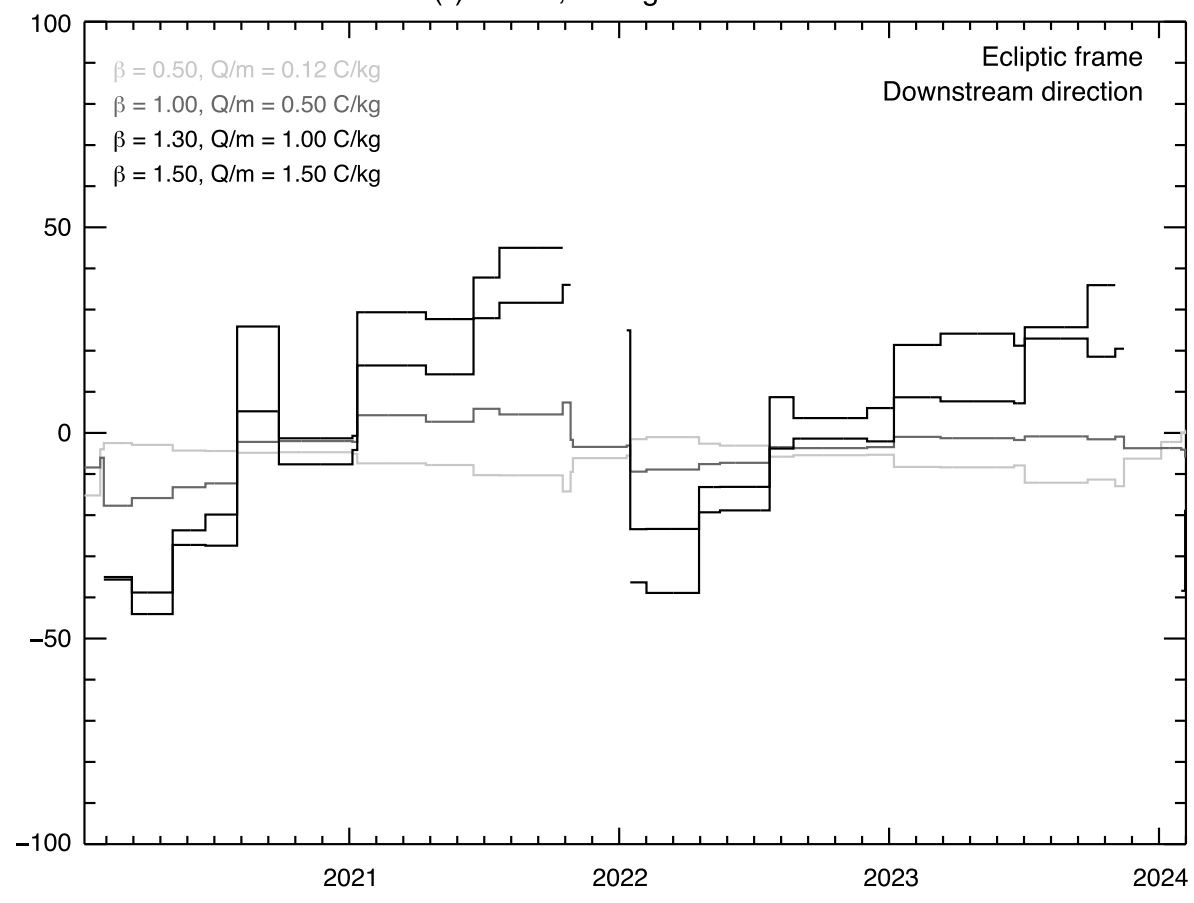

Fig. 9 Directionality variation (ecliptic latitude) of the interstellar dust direction of SARIM PLUS. Only small particles show deviations greater than $30^{\circ}$ from the interstellar latitude of $-8^{\circ}$ 
is deduced and measurements outside of the cone will link grain directionalities to certain $\beta$ and $\mathrm{Q} / \mathrm{m}$ values.

Figures 8 and 9 show the predicted directionality for the same set of particles as in Table 3. The relative velocity of SARIM PLUS with respect to the ISD velocity is high in the years of 2020-2021 and 2022-2023, whereas in the periods of 2021-2022 and 2023-2024 the relative velocity is low. This raises or lowers the expected flux, which is visible through the slope of the flux curve segments. The maximum relative velocity occurs in mid-2020 and mid-2022 (approximately $57 \mathrm{~km} / \mathrm{s}$ for dust with $\beta=1, \mathrm{Q} / \mathrm{m}=0$ ), while the minimum relative velocity is in mid-2021 and mid-2023 (appr. $7 \mathrm{~km} / \mathrm{s}$ for dust with $\beta=1, \mathrm{Q}=0$ ).

\section{Payload}

The scientific payload shall allow in-situ dust and plasma measurements as well as collection of IDP, ISD and cometary dust. Furthermore, imaging is crucial for navigation and the cometary encounter science. This chapter discusses payload components which are suited for SARIM PLUS and which were part of former mission concepts like SARIM or DuneXpress. They do not represent a formal model payload yet due to the recent developments and activities in the field of active dust collectors (e.g. Univ. Boulder), collector developments (USA, Jp) and dust spectrometers (Ger, F, Jp) or robotic concepts (for collector handling). A camera shall be part of the instruments for comet coma and nucleus imaging, but is not described here. Furthermore, a return capsule is required on the basis of Stardust (almost same reentry speed), but is also not described.

The different mission phases (high relative impact speeds ISD, low relative impact speeds of ISDs, IDP pointing, comet encounter) require distinct collector modules. Therefore more than one collector is required in contrast to Stardust. Individual collectors shall be handled by a robotic mechanism to pull them from the storage container for exposure. A preliminary payload concept was developed for the SARIM payload described in Srama et al. [44]. It consists of seven Active Dust Collectors, one Dust Telescope, one Nanoparticle detector, a plasma monitor and one Sample Return Capsule (SRC). A nano-particle detector is a new type of detector with an increased sensitivitysuch a detector was never flown on interplanetary spacecraft. Such a payload allows a sensitive and reliable determination of individual dust grain characteristics as speed, mass, charge, trajectory and composition. The combination of in-situ and sample return techniques is unique and provides cross-calibration processes during and after the mission.

\subsection{Active dust collector}

A preliminary collector concept uses seven dust collector modules of a size of $400 \mathrm{~mm} \times 400 \mathrm{~mm} \times 20 \mathrm{~mm}$ with a total mass of $0.5 \mathrm{~kg}$ per collector plate plus supporting structure. The capsule contains a robotic arm to place the 
Fig. 10 Active Dust

Collector with Trajectory Sensor, movable collector plate and impact detector

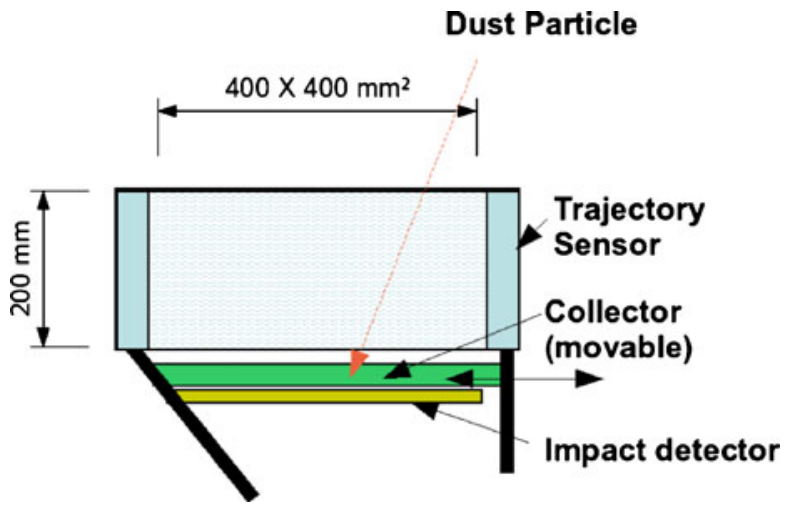


An Active Dust Collector is a combination of an in-situ Dust Trajectory Sensor (DTS) together with a dust collector consisting of aerogel or other collector materials, e.g. like the ones used on the STARDUST mission. Dust particles' trajectories are determined by the measurement of induced electric signals when charged grains fly through a position sensitive electrode system [29]. The recorded waveforms allow us to reconstruct the velocity vector with high precision. Such a DTS was subject to performance tests at the Heidelberg dust accelerator. At the same time impact signals from potential collector materials were recorded. These tests with dust particles in the speed range from 3 to $40 \mathrm{~km} / \mathrm{s}$ demonstrate that trajectories can be measured with accuracies of $\sim 1^{\circ}$ in direction, and $\sim 1 \%$ in speed. The sensor measures dust charges of a few $10^{-16} \mathrm{C}$ and, thereby, provides trajectory information of collected cosmic dust particles as small as $0.3 \mu \mathrm{m}$ in radius and facilitates the determination of the impact position on the collector to better $1 \mathrm{~mm}$ precision which will immensely ease the task of finding sub-micron sized particles on the collector. In interplanetary space statistically significant numbers of trajectories of interplanetary and interstellar dust grains can thus be collected and their compositions correlated with their interplanetary trajectories.

Especially during cruise periods of low relative velocity the ISD collectors are exposed and will be located between the Dust Trajectory Sensor (DTS) and the impact detector by a robotic mechanism (Fig. 11). Once the collector is removed, the dust grains pass the Trajectory Sensor and impact onto the impact detector (Fig. 10). The impact stage itself is a separate dust sensor applying a variety type of detection techniques to get a complementary information about the dust grains. Detection techniques used here are either impact ionisation (used by 5 modules), depolarisation of foils (1 module) or acoustic signals (1 module). By the means of seven active collector modules, a concept as shown in Table 4 can be applied.

All modules have Dust Trajectory Sensors (DTS) in front of the collector. A simple square trajectory sensor consists of four sensor grids mounted between two electrical shielding grids [41, 42]. The distance between grid planes is $40 \mathrm{~mm}$. Each sensor grid consists of 15 parallel wire electrodes (wires separated by $20 \mathrm{~mm}$ ), each electrode has a capacitance of about $5 \mathrm{pF}$ and is connected to a separate charge sensitive amplifier (CSA). The wire directions of adjacent sensor grids are orthogonal. Each pair of adjacent wire electrodes within a sensor grid acts as a one-dimensional position-sensitive detector. Key elements

Table 4 Module concept of the SARIM PLUS payload

\begin{tabular}{lll}
\hline Module number & Collector type & Impact detector \\
\hline 1 & Aerogel & Dust Camera 1 \\
2 & Aerogel & Dust Camera 2 \\
3 & Aerogel & Dust Camera 3 \\
4 & Foil Type 1 & Dust Camera 3 \\
5 & Foil Type 2 & Dust Camera 3 \\
6 & Foil Type 3 & Dust Camera 3 \\
7 & Foil Type 4 & Dust Camera 3 \\
\hline
\end{tabular}


of the trajectory sensor are the charge-sensitive amplifier (CSA) and the transient recorder.

The collector modules are either frames with aerogel with a low density, or frames with ultra-clean foils. In contrast to aerogel collectors used by the Stardust mission, newly developed materials (metal foams or black materials) are also suited to analyse silicate components of dust grains. Furthermore, new preparation techniques allow a ten-times cleaner fabrication which is mandatory for the analysis of organic components.

The selection of the collector materials has to be done in accordance with the latest knowledge and Stardust results. For deep-space collection of interplanetary (asteroidal and cometary) and interstellar particles (dominated by silicate/sulfide/oxide minerals with very small grains including amorphous, non-crystalline materials, diamond, exotic carbides and nitrides), the ideal substrate must not contain elements likely to be of major significance in captured particles ( $\mathrm{Na}, \mathrm{Mg}, \mathrm{Al}, \mathrm{Si}, \mathrm{P}, \mathrm{S}, \mathrm{K}, \mathrm{Ca}, \mathrm{Ti}, \mathrm{Cr}, \mathrm{Mn}, \mathrm{Fe}, \mathrm{Ni}$ ). H, C, N and $\mathrm{O}$ should be absent, or their isotopic composition in the substrate should be highly distinctive if they are to be distinguished from captured organic material.

The collection medium should: (1) capture any particle between $10 \mathrm{~nm}$ and $1 \mathrm{~mm}$ size, regardless of particle structure, mass or velocity (2) result in lowest possible structural or compositional modification during or subsequent to capture (3) allow rapid and unambiguous location of all captured particles on return to the laboratory (4) give no interference with in situ analysis techniques, and no ambiguity as to the origin of specific elemental signatures permit perfect physical separation of the captured particle prior to further, sophisticated analysis. Criteria for assessment of suitability for potential collector substrates are:

(a) The amount of material retained on the collector. Brittle materials spall extensively, resulting in sample loss. Ductile materials may deform, but retain more of the impacting particle.

(b) The extent of particle processing during capture, due to the peak pressures and temperatures attained (primarily a function of substrate density) and sustained (controlled by thermal conductivity of the substrate), including both changes to the structural state of the particle (shock deformation of a crystal lattice, melting or partial/entire loss as vapour) during and after capture, and compositional modification due to mixing with other impactor components, the substrate or by loss to vapour.

(c) The medium composition should be simple and pure enough that it can be distinguished from all important particle components without ambiguity.

(d) The ease of application of primary in-situ survey and analysis methods to collector components made of this material, including whether, after sample acquisition, the material can be handled easily as large areas without damage or contamination, and can be examined in suitable instrumentation without need for destructive preparation in early stages of study. 
(e) The ease of clean, uncontaminated particle extraction for further analysis, for separation from the substrate and subsequent examination in sophisticated instruments.

(f) Methods used for subsequent measurement of particle/residue structure and composition: IR and Raman spectroscopy, synchrotron IR, XRD, XRF, XANES, analytical FIB-TEM, SIMS, ToF-SIMS and NanoSIMS, bearing in mind their differing needs for cleanliness, sample size and analytical substrates.

At present no material meets all of the desirable criteria perfectly. However, three of them probably provide the best available compromise: silica aerogel [7], polymer and metal foil surfaces [16, 19, 23-28], albeit in modified form to those deployed on previous missions, and each with different limitations.

\subsection{Dust Cameras and instrumentation}

Dust Cameras are combined Trajectory Sensors and Impact Detectors without the collector plate inbetween (Table 5). During non-collection phases, the impact detector will trigger the instrument and derives particle momentum, mass and impact location. Complementary impact detectors shall be used in order to contrain the dust particle properties. Three different dust cameras are proposed and they are described in the SARIM and DuneXpress mission concepts $[18,44]$.

Dust Camera 1 uses polyvinylidene fluoride (PVDF) film to determine the mass of a dust particle passing through a Trajectory Sensor. PVDF was developed as a dust impact sensor and flown successfully on various missions like Stardust or Cassini $[39,40,50]$. This instrument comprises 25 discrete (in a $5 \times$ 5 array) PVDF film modules, each with approximate dimensions $6 \mathrm{~cm} \times 6 \mathrm{~cm}$. Using 25 modules minimizes capacitance of each sensor element (Fig. 12), as well as aiding reliability and discrimination between impact sites. The PVDF instrument contributes less than $3 \mathrm{~kg}$ and $2 \mathrm{~W}$ to the total mass/power budget of the whole camera.

The impact/momentum sensor Dust Camera 2 (DC2) is based on the use of the impact sensor concept, developed e.g. for the GIADA instrument onboard Rosetta mission. The detector plate is equipped with piezoelectric sensors and their number is adequate to have multiple measurements of a single impact, which give impact position and an intrinsic redundancy in the sensor [14]. The impact sensor is capable of providing information about the flux of particles encountered and about a combination of their mass and speed, very closely related to the momentum. If the relative velocity of the sensor with respect to the local environment is measured by the trajectory sensor (or is known in the case of a cometary flyby), the mass of each particle can be derived.

The impact sensor plate developed for previous experiments (e.g., GIADA) has a $0.5 \mathrm{~mm}$ thick aluminium diaphragm and square sensitive area $0.01 \mathrm{~m}^{2}$. 


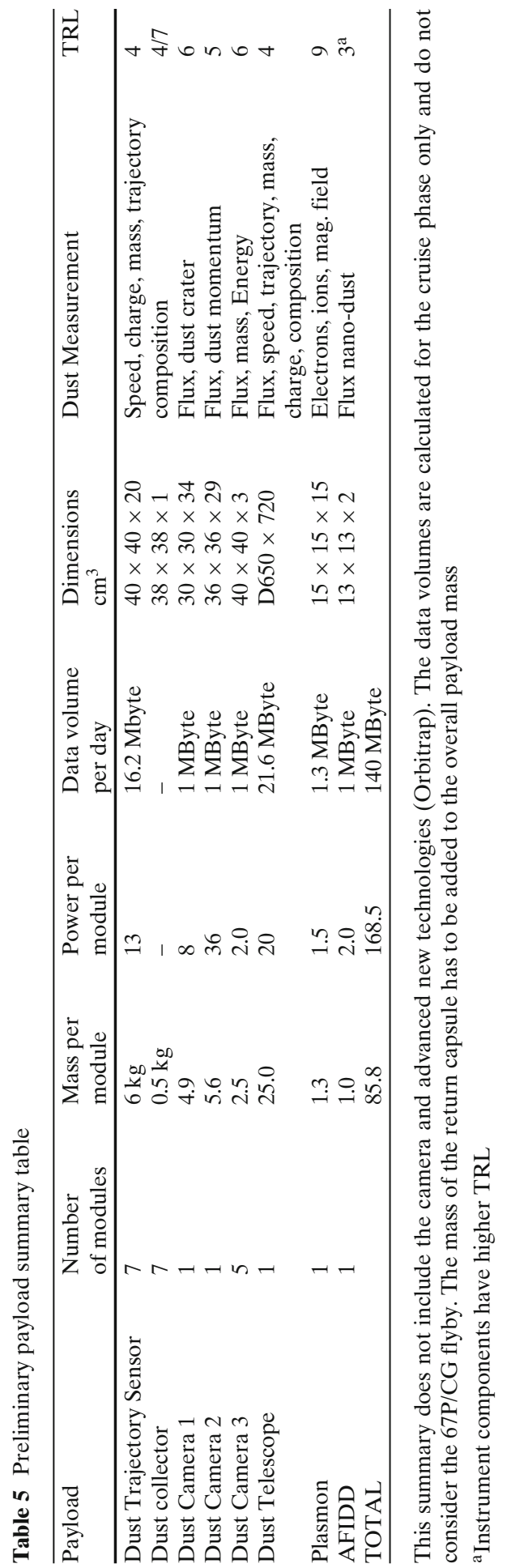


Fig. 12 PVDF film modules of Dust Camera 1

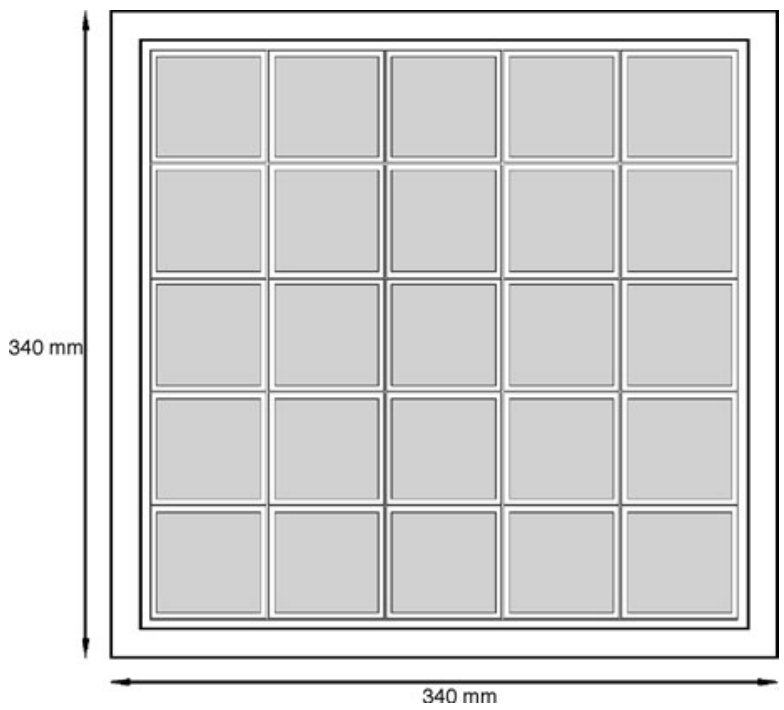

DC2 shall have a sensitive area of $0.1 \mathrm{~m}^{2}$, which can be guaranteed by integrating several parallel modules or by developing a larger module. The sensors detect a minimum momentum of $6.5 \times 10^{-10} \mathrm{~kg} \mathrm{~m} \mathrm{~s}^{-1}$. The saturation limit is expected at a momentum of $4.0 \times 10^{-4} \mathrm{~kg} \mathrm{~m} \mathrm{~s}^{-1}$. The detection limit corresponds to mass sensitivity of $1.3 \times 10^{-13}, 6.5 \times 10^{-14}$ and $3.2 \times 10^{-14} \mathrm{~kg}$ for 5,10 and $20 \mathrm{~km} \mathrm{~s}^{-1}$ relative speed [10-13].

Dust Camera 3 uses impact ionization upon a hyper-velocity impact of a dust grain at a target plate. In situ data from such instruments have been obtained over several decades (Pioneer, Helios, Galileo, Ulysses, Cassini). In this instrument, a particle impacts a flat target plate $($ at $0 \mathrm{~V})$, and impact cations are accelerated towards an electrode grid (at $-100 \mathrm{~V})$. The total charge collection at this electrode is related to the particle mass and velocity. As velocity is independently determined by the Trajectory Sensor, the mass of the particle can thus be obtained. The instrument comprises 25 sensing modules, each $9 \mathrm{~cm} \times 9 \mathrm{~cm}$, mounted in a $5 \times 5$ array similar to the PVDF-based camera. This instrument contributes less than $4 \mathrm{~kg}$ and $2 \mathrm{~W}$ to the total mass/power budget of the whole camera.

The Dust Telescope (DT) combines a Trajectory Sensor with an analyser for the elemental composition of micrometeoroids [42, 47]. The combination of two subsystems to one high-performance detector allows for a simultaneous determination of the dust properties mass, velocity vector, surface charge and, with high mass resolution, dust composition.

The large area mass analyser (LAMA) is based upon impact ionisation of hyper-velocity dust impacts onto a ring shaped target plate. This time-of-flight system uses a reflectron for an increased mass resolution and provides the elemental composition of individual micrometeoroids with a mass resolution 
between 100 and 300 [40, 41]. Although a similar instrument was already flown onboard Stardust [30], the Dust Telescope has a sensitive area 10 times larger. A decontamination heater at the target is operated every 3 months and will ensure a clean surface of the impact target.

Latest developments in spectrometry allow the integration of a novel Orbitrap system within a Dust Telescope or linear spectrometers by using ringshaped MCPs and/or focusing electrodes [22]. The Orbitrap ion detector is a small mass spectrometer itself and provides mass resolutions beyond 10.000 which is unique in space applications and which is requested by astrobiologists. Complex organic molecules during the comet flyby and of individual interstellar grains are characterised with high accuracy. Orbitrap is just $7 \mathrm{~cm} \times 3 \mathrm{~cm}$ big and is currently adjusted to space applications by CNRS [48].

In order to extend the measurement window to nano-metre sized micrometeoroids, an aluminium Film Interplanetary Dust Detector (AFIDD) as a Nano-Dust Detector is required [8,9]. Dust particles impacting on a thin $(10-$ $100 \mathrm{~nm}$ ) Al film will have a ballistic limit defined as the maximum thickness of aluminium which can be perforated [33]. The limit is a function of both the mass and velocity of a particle and is derived from empirical formulae generated from laboratory impact data [15]. AFIDD consists of an array of four circular, Al filmed MCP detectors bearing two film thicknesses $(2 \times 10 \mathrm{~nm}$ and $2 \times 100 \mathrm{~nm}$ ). The $\mathrm{Al}$ films are supported by the interchannel walls of an MCP detector and are freestanding over the open area of the microchannels. All MCPs share common electronics, casing and pointing direction. The MCP detectors are operated in a "low-gain" mode such that the pulse height becomes a function of the total energy deposited in the microchannel and can therefore be used to discriminate between dust and radiation induced events. AFIDD will detect hypervelocity impacts dust particles with masses of the order of $10^{-18} \mathrm{~g}$ and less.

A magnetometer, an electrostatic plasma analyser and a Faraday cup are combined in a $300 \mathrm{~g}$ spherical PLASMON sensor [18]. Using channeltrons in a counting mode, a small electrostatic plasma analyser measures the electron and ion distribution in a wide energy range. Hemispherical deflection plates are used to analyse the energy distribution in 32 steps. Assuming a radial solar wind bulk velocity, all major plasma parameters such as electron and proton densities, temperatures, and proton bulk velocities can be derived. The energy ranges measured are 40-8000 eV (ions) and $0.35-4200 \mathrm{eV}$ (electrons). The Faraday cup measures ions up to $2 \mathrm{keV}$. The magnetic field is measured with a vector compensated ringcore fluxgate magnetometer. The resolution of the magnetometer $(10 \mathrm{pT})$ is only restricted by the sensor noise $\left(<5 \mathrm{pT} \mathrm{Hz}^{-0.5}\right.$ at $1 \mathrm{~Hz}$ ) and fields up to $1000 \mathrm{nT}$ can be determined.

Key technology areas are the re-entry capsule development, the robotic mechanism for the collector handling and storage, the Dust Telescope (ion to electron converter), a possible Orbitrap spectrometer integration and the ASIC development for the Trajectory Sensor. 


\section{Conclusions}

Stardust returned cometary, interplanetary and (probably) interstellar dust to Earth in 2006. Rosetta will investigate in great detail the comet 67P/Churyumov Gerasimenko starting in 2014. Further missions will contribute to our understanding of the overall picture of dust generation, evolution and destruction, which is important to understand solar system formation and the origin of life. SARIM-PLUS will link this knowledge by measuring interplanetary and interstellar dust with high accuracy and sensitivity in our inner solar system between 1 and 2 AU solar distance. It employs latest insitu techniques for a full characterisation of individual micrometeoroids (flux, mass, charge, trajectory, composition) and collects and returns samples to Earth for a detailed analysis. The opportunity to visit again the target comet of the Rosetta mission, 67P/CG, and to investigate its dusty environment six years after Rosetta with complementary methods is unique and strongly enhances the scientific output of the Rosetta mission.

Launch opportunities are in 2020 with a backup window starting early 2026. The comet encounter occurs in September 2021 and the reentry takes place in early 2024. The encounter speed of $6 \mathrm{~km} / \mathrm{s}$ ensures comparable results to the Stardust mission.

SARIM-PLUS performs both, in-situ measurements and sample return of interplanetary micrometeoroids, interstellar (ISD) and cometary dust. The overall mission duration of 4 years allows for sufficient collection time and in-situ measurements during cruise. Stardust was optimised for the collection of cometary dust, SARIM-PLUS will be optimised for both the collection of ISD and of cometary dust taking into account the results of Rosetta, Stardust, Deep Impact and other missions.

\section{References}

1. Altobelli, N., et al.: Cassini between Venus and Earth: detection of interstellar dust. J. Geophys. Res. 108(A10), 8032 (2003)

2. Altobelli, N., et al.: A new look into the Helios dust experiment data: presence of interstellar dust inside the Earth orbit. Astron. Astrophys. 448, 243-252 (2006)

3. Altobelli, N., et al.: Interstellar dust flux measurements by the Galileo dust instrument between the orbit of Venus and Mars. J. Geophys. Res. 110, 7102 (2005)

4. Baggaley, W.J.: Advanced meteor orbit radar observations of interstellar meteoroids. J. Geophys. Res. Space Phys. 105(A5), 10353-10361 (2000)

5. Brownlee, D.E., et al.: Stardust: comet and interstellar dust sample return mission. J. Geophys. Res. 108(E10), SRD 1-1 (2003)

6. Brownlee, D.E.: 164 co-authors: Comet Wild 2 under a microscope. Science 314, 1711-1716 (2006)

7. Burchell, M.J., et al.: Cosmic dust collection in aerogel. Ann. Rev. Earth Planet. Sci. 34, 385$418(2006)$

8. Carpenter, J.D., et al.: Dust detection in the ISS environment using filmed microchannel plates. J. Geophys. Res. 110, E05013 (2005) 
9. Carpenter, J.D., Stevenson, T.J., Fraser, G.W., Bridges, J.C., Kearsley, A.T., Chater R.J., Hainsworth, S.V.: Nanometer hypervelocity dust impacts in low Earth orbit. J. Geophys. Res. 112, E08008 (2007). doi:10.1029/2007JE002923

10. Colangeli, L., et al.: Preface to the special section: space simulations in laboratory: experiments, instrumentation, and modeling. J. Geophys. Res. 109(E07S01), 1-2 (2004). doi:10.1029/2004JE002292

11. Colangeli, L., et al.: The new Rosetta targets-observation, simulations and instrument performances. Astrophys Space Sci. Library 311, 271-280 (2004)

12. Colangeli, L., et al.: GIADA: the grain impact analyser and dust accumulator for the Rosetta space mission. Adv. Space Res. 39(3), 446-450 (2007)

13. Colangeli, L., et al.: The Grain Impact Analyser and Dust Accumulator (GIADA) Experiment for the Rosetta Mission: design, performances and first results. Space Sci. Rev. 128, 803-821 (2007)

14. Esposito, F., et al.: Physical aspect of an "impact sensor" for the detection of cometary dust momentum onboard the "Rosetta" space mission. Adv. Space Res. 29, 1159-1163 (2002)

15. Gardner, D.J., et al.: Hole growth characterisation for hypervelocity impacts in thin targets. Int. J. Impact Eng. 19(7), 589-602 (1997)

16. Graham, G.A., et al.: Observations on hypervelocity impact damage sustained by multilayered insulation foils exposed in low earth orbit and simulated in the laboratory. Int. J. Impact Eng. 29, 307-316 (2003)

17. Grün, E., Gustfason, B., Mann, I., Baguhl, M., Morfill, G.E., Staubach, P., Taylor, A., Zook, H.A.: Interstellar dust in the heliosphere. Astron. Astrophys. 286, 915-924 (1994)

18. Grün, E., et al.: DuneXpress. Exp. Astron. 23(3), 981 (2009). doi:10.1007/s10686-008-9099-4

19. Hoppe, P., et al.: SIMS studies of Allende projectiles fired into Stardust-type aluminium foils at $6 \mathrm{~km} / \mathrm{sec}$. Meteorit. Planet. Sci. 41.2, 197-209 (2006)

20. Hoppe, P.: Origin and early evolution of comet nuclei. Space Sci. Series ISSI 28, 43-57 (2009)

21. Hörz, F., et al.: Impact features on Stardust and Comet Wild 2 Dust. Science 314, 1716-1719 (2006)

22. Hu, Q., et al.: The Orbitrap: a new mass spectrometer. J. Mass Spectr. 40, 430-443 (2005)

23. Kearsley, A.T., Graham, G.A.: Multi-layered foil capture of micrometeoroids and orbital debris in low Earth orbit. Adv. Space Res. 34, 939-943 (2004)

24. Kearsley, A.T., et al.: Impacts on Hubble Space Telescope solar arrays: discrimination between natural and man-made particles. Adv. Space Res. 35, 1254-1262 (2005)

25. Kearsley, A.T., et al.: Sampling the orbital debris population using a foil residue collector in a Standardised Container For Experiments (SCE). In: Danesy, D. (ed.) Proceedings of the 4th European Conference on Space Debris, ESA Special Publication 587. The European Space Agency, Darmstadt, Germany, pp. 215-220 (2005)

26. Kearsley, A.T., et al.: MULPEX: a compact multi-layered polymer foil collector for micrometeoroids and orbital debris. Adv. Space Res. 35, 1270-1281 (2005)

27. Kearsley, A.T., et al.: Analytical scanning and transmission electron microscopy of laboratory impacts on Stardust aluminum foils: interpreting impact crater morphology and the composition of impact residues. Meteorit. Planet. Sci. 42.2, 191-210 (2007)

28. Kearsley, A.T., et al.: Dust from comet Wild 2: interpreting particle size, shape, structure and composition from impact features on the Stardust aluminum foils. Meteorit. Planet. Sci. (2007, submitted)

29. Kempf, S., et al.: Cassini between Earth and asteroid belt: discovery of charged interplanetary dust grains. Icarus 171(2), 317-335 (2004)

30. Krueger, F.R., Werther, W., Kissel, J., Schmid, E.R.: Assignment of quinone derivates as the main compound class composing interstellar grains based on both polarity ions detected by the Cometary and Interstellar Dust Analyser (CIDA) onboard the spacecraft STARDUST. Rap. Comm. Mass Spec. 18, 103-111 (2004)

31. Kuan Y.-J., et al.: A search for interstellar pyrimidine. Monthly Notices of the Royal Astronomical Society 345(10), 650-656 (2003)

32. Landgraf, M., et al.: Aspects of the mass distribution of interstellar dust grains in the solar system from in situ measurements. J. Geophys. Res. 105, 10343 (2000) 
33. McDonnell, J.A.M., et al.: Near Earth environment. In: Grun, E. (ed.) Interplanetary Dust, pp. 163-261. Springer, London and Berlin (2001)

34. McKeegan, K.D., et al.: Isotopic compositions of cometary matter returned by Stardust. Science 314, 1724-1728 (2006)

35. Mumma, M.J.: Organic volatiles in comets: their relation to interstellar ices and solar nebula material, from stardust to planetesimals. Symposium held as part of the 108th Annual meeting of the ASP held at Santa Clara, California 24-26 June 1996. ASP Conference Series, vol. 122, pp. 369 (1997)

36. Nesvorny, D., Jenniskens, P., Levison, H.F., Bottke, W.F., Vokrouhlický, D., Gounelle, M.: Cometary origin of the zodiacal cloud and carbonaceous micrometeorites - implications for hot debris disks. Astrophys. J. 713, 816-836 (2010)

37. Sandford, S.A., et al.: Organics captured from comet 81/PWild 2 by the Stardust spacecraft. Science 314, 1720 (2006)

38. Slavin, J.D., Frisch, P.C.: Exclusion of tiny interstellar dust grains from the heliosphere. Astron. Astrophys. 491, 53-68 (2008)

39. Simpson, J.A., Tuzzolino, A.J.: II.: Instruments for measurement of particle trajectory, velocity and mass. Nucl. Inst. Meths. Sect. A 279, 611-624 (1989)

40. Srama, R., et al.: The Cassini cosmic dust analyser. Space Sci. Rev. 114, 465-518 (2004)

41. Srama, R., et al.: Performance of an advanced dust telescope. In: Danesy D. (ed.) Proceedings of the 4th European Conference on Space Debris (ESA SP-587), p. 171. ESA/ESOC, 18-20 April 2005. Darmstadt, Germany (2005)

42. Srama, R., et al.: Development of an advanced dust telescope. Earth Moon Planets 95(1-4), 211-220 (2005). doi:10.1007/s11038-005-9040-z

43. Srama, R., Kempf, S., Moragas-Klostermeyer, G., Landgraf, M., Helfert, S., Sternovsky, Z., Rachev, M., Grün, E.: Laboratory tests of the large area mass analyzer. In: Proc. of "Dust in Planetary Systems", pp. 209-213. ESA SP-643, Kauai, Hawaii, USA (2007)

44. Srama, R., et al.: Sample return of interstellar matter (SARIM). Exp. Astron. (2008). doi:10.1007/s10686-008-9088-7

45. Sterken, V.J., Altobelli, N., Kempf, S., Schwehm, G., Srama, R., Grün, E.: The flow of interstellar dust into the Solar System. Astron. Astrophys (2011, in press)

46. Stephan, T., et al.: TOF-SIMS analysis of cometary matter in Stardust aerogel tracks. Meteorit. Planet. Sci. 43(1-2), 233-246 (2008)

47. Sternovsky, Z., Amyx, K., Bano, G., Landgraf, M., Horanyi, M., Knappmiller, S., Robertson, S., Grün, E., Srama, R., Auer, S.: The Large Area Mass Analyzer (Lama) instrument for the chemical analysis of interstellar dust particles. Rev. Sci. Instrum. 78, 014501 (2007)

48. Thissen, R.: person. communication (2010)

49. Tsou, P., et al.: Wild 2 and interstellar sample collection and Earth return. J. Geophys. Res. (2003). doi:10.1029/2003JE002109

50. Tuzzolino, A.J., et al.: Dust Flux Monitor Instrument for the Stardust mission to comet Wild 2. J. Geophys. Res. 108(E10), 8115 (2003). doi:10.1029/2003JE002086

51. Westphal, A.J., Fakra, S.C., Gainsforth, Z., Marcus, M.A., Ogliore, R.C., Butterworth, A.L.: Mixing fraction of inner solar system material in comet 81P/Wild 2. Astrophys. J. 694, 1 (2009)

52. Westphal, A., et al.: LPSC 41, 2050 (2010)

53. Witte, M., et al.: Astron. Astrophys. 426, 835 (2004)

54. Zolensky, M.E., et al.: Mineralogy and petrology of Comet 81P/Wild 2 nucleus samples. Science 314(5806), 1735 (2006) 